This document is published in:

Oral Surgery, Oral Medicine, Oral Pathology and Oral Radiology, Vol. 113, no 5 (2012), pp. 4-11

DOI: http://dx.doi.org/10.1016/j.tripleo.2011.07.030

(C) 2012 Elsevier Inc. 


\section{Clinical outcomes after the use of complete autologous oral mucosa equivalents: preliminary cases}

Ignacio Peña, DDS, MD, PhD, ${ }^{a}$ Luis Manuel Junquera, MD, DDS, PhD, ${ }^{\text {a }}$ Santiago Llorente, MD, DDS, PhD, ${ }^{\text {b }}$ Lucas de Villalaín, MD, DDS, PhD, ${ }^{a}$ Juan Carlos de Vicente, MD, DDS, PhD, ${ }^{\mathrm{a}}$ and Sara Llames, PhD, ${ }^{\mathrm{c}}$

Central University Hospital of Asturias, University of Oviedo, and Blood and Tissues Community Center of Asturias, Oviedo, Spain

Objective. Previously, we reported how to obtain complete autologous oral mucosa equivalents (CAOMEs) composed of an autologous plasma scaffold and fibroblasts together with immature keratinocytes able to build an oral epithelium with a structure similar to that of the oral mucosa. In this study, we present the clinical outcomes after applying our CAOMEs as grafts.

Study Design. Four patients who needed a CAOME to restore a defect of oral mucosa were selected. Two of the patients suffered from ankyloglossia, and the other 2 required a restoration of the keratinized gum of the alveolar rim. To assess the outcomes, the scale designed by Ewers et al. was used.

Results. Clinical and functional improvements were achieved in the patients with ankyloglossia. In cases of gum restoration, the mucosa was regenerated and a prosthetic restoration with implants was achieved.

Conclusions. The results obtained points to the potential use of CAOME in intraoral lining.

The purpose of oral mucosa equivalents developed in vitro is to repair both congenital (such as labial-palatal clefts) and acquired (due to traumatic or pathologic causes) defects. These types of techniques can be widely used beyond the oral field examples of additional applications include repairing the cornea, ${ }^{1-3}$ the palpebral conjunctiva, the nasal mucosa, the esophagus, the trachea, the urethra, and the vagina, ${ }^{4}$ and they can also be used as an alternative to skin culture for major burn coverage. ${ }^{5}$ Several authors have applied these techniques to the reconstruction of the oral mucosa with uneven clinical results. ${ }^{6-8}$

Previously, we reported how to obtain complete autologous oral mucosa equivalents (CAOMEs) composed of an autologous plasma scaffold and fibroblast together with immature keratinocytes able to build an oral epithelium with a structure similar to that of the oral mucosa. ${ }^{9}$

In the present study, we discuss the CAOME-based treatment applied to 4 patients who required a recon-

Supported by a grant from the Sanity Institute Carlos III (Spanish Government).

${ }^{\mathrm{a}}$ Oral and Maxillofacial Surgeon, Central University Hospital of Asturias; Department of Surgery and Dental College, University of Oviedo.

${ }^{\mathrm{b}}$ Oral and Maxillofacial Surgeon, private practice.

${ }^{\mathrm{c} B}$ lood and Tissues Community Center of Asturias. struction of the oral mucosa. The objective was twofold: to assess as objectively as possible whether the patients exhibit a clinical improvement and to determine if our technique reduced the healing time reported in the literature when no medium is used to cover the defect.

\section{MATERIALS AND METHODS}

\section{CAOME Culture and Engineering}

A biopsy from healthy oral mucosa $(5 \times 5 \mathrm{~mm})$ and a $27-\mathrm{mL}$ blood sample were taken from each patient. The samples were divided into small fragments and subjected to 4 washes with $5 \mathrm{~mL}$ phosphate-buffered saline solution (PBS) containing trypsin/EDTA (Gibco, Invitrogen) at a concentration of $0.05 \% / 0.02 \%$, respectively. The resulting cell pellet was used to obtain the primary keratinocyte culture. The remaining tissue fragments were subjected to a fift wash with collagenase (Sigma) and DMEM at a concentration of $2 \mathrm{mg} / \mathrm{mL}$, to obtain the cell pellet to be used for the primary fibroblas culture.

The keratinocyte growth medium was QN [DMEM (Gibco, Invitrogen) and Ham F-12 (Gibco, Invitrogen) in a $3: 1$ ratio $+10 \%$ fetal bovine serum (FBS; Gibco, Invitrogen $)+$ insulin $(5 \mathrm{~g} / \mathrm{mL}$; Sigma $)+$ cholera toxin $(8 \mathrm{ng} / \mathrm{mL}$; Sigma $)+$ adenine $(24 \mathrm{mg} / \mathrm{mL}$; Sigma $)+$ triiodothyronine $(1.3 \mathrm{ng} / \mathrm{mL}$; Sigma) + hydrocortisone $(0.4 \mu \mathrm{g} / \mathrm{mL}$; Sigma) $]$ and required the use of lethally irradiated 3T3 cells as a feeder layer. For the cultivation of fibroblasts DMEM supplemented with 10\% FBS was used. After 3 days of culture, epidermal growth factor (10 ng/mL EGF; Austral Biologicals, San Ra- 
Table I. Pathologies before surgery, the surgical sequelae, and the type of oral mucosa the patients needed

\begin{tabular}{cllc}
\hline Patient & \multicolumn{1}{c}{ Pathology } & \multicolumn{1}{c}{ Sequel } & Mucosa required \\
\hline 1 & Epidermoid carcinoma stage II & Ankyloglossia & Nonkeratinized \\
2 & Epidermoid carcinoma stage II & Ankyloglossia & Nonkeratinized \\
3 & Pleomorphic adenoma & Absence of alveolar gum & Keratinized \\
4 & Epulis fissuratu & Absence of alveolar gum & Keratinized \\
\hline
\end{tabular}

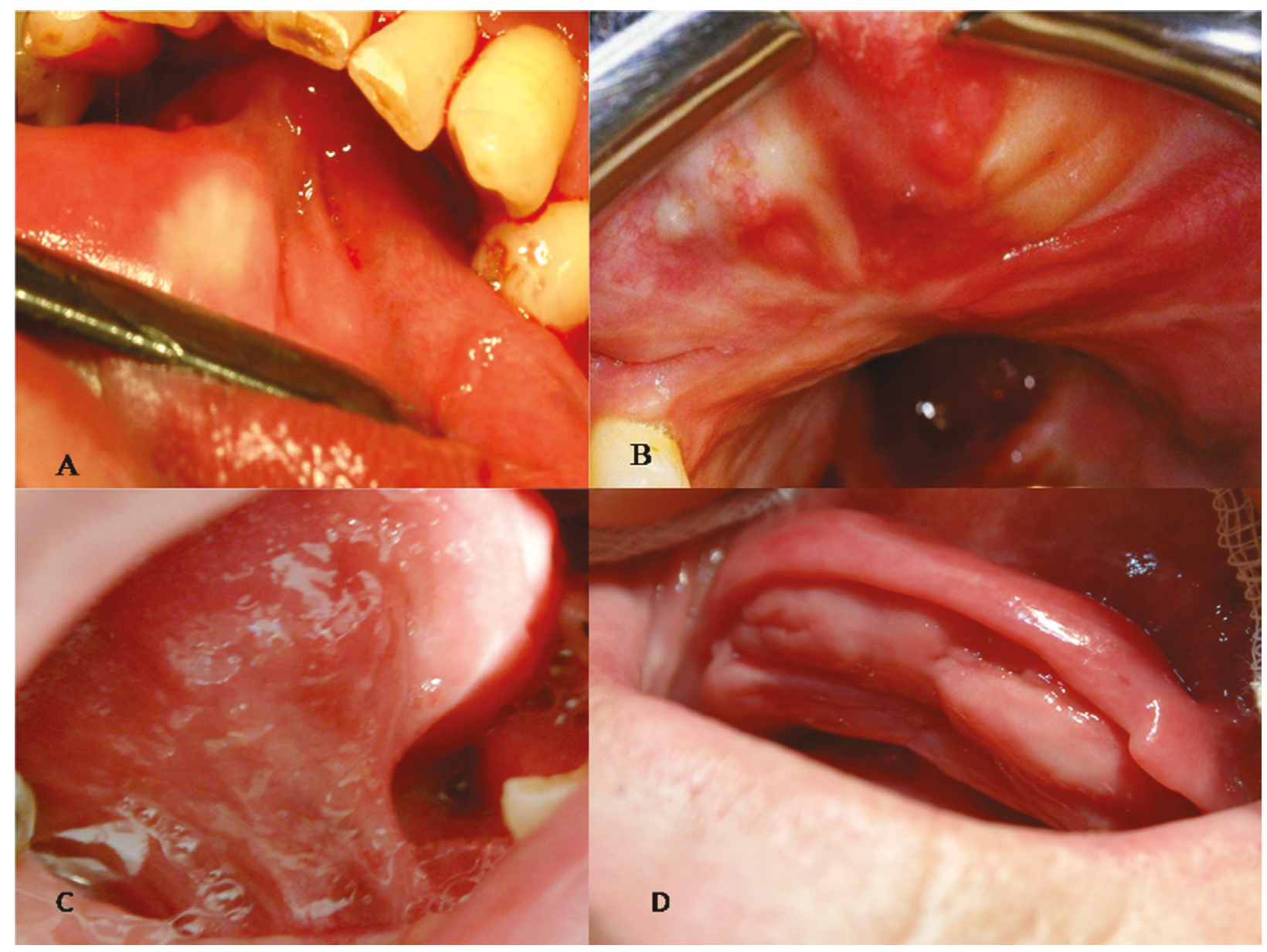

Fig. 1. A, Patient 1, with a scar bridle definin an ankyloglossia, with speech impediment. B, Patient 3, with reduced vestibular depth, requires keratinized gum and an expansion of the prosthetic space by removing the existing oral mucosa flap C, Patient 2, exhibiting a bridle that affects most of the right side lingual rim and oral floor hindering phonation and lingual motility. D, Patient 4, featuring an epulis fissuratu that would require keratinized gum after resection; therefore, a complete autologous oral mucosa equivalent grafting was scheduled during the same surgical act after lesion resection.

mon, CA) was added to the keratinocyte culture. When cells reached $90 \%$ confluence a firs pass (P1) was made into T-25 flask for keratinocytes and T-12.5 flask for fibroblasts Time for P1 keratinocytes until confluenc was 7-8 days. During that period a second pass (P2) was needed for fibroblast into T-75 flasks We used fibri glue obtained from the patient's blood sample as scaffolds. To construct a $25-\mathrm{cm}^{2}$ CAOME, we used $4 \mathrm{~mL}$ plasma, $22 \times 10^{3}$ fibroblasts $0.7 \mathrm{~mL}$ calcium chloride at $1 \%$ in saline serum, $70 \mu \mathrm{L}$ tranexamic acid (Fides-Ecofarma, Almacera, Spain), and saline serum. The mixture was then allowed to solidify at $37^{\circ} \mathrm{C}$ for $30-60$ minutes with $4.7 \mathrm{~mL}$ QN medium. After that period, keratinocytes were seeded. Fibroblasts were from P2 and keratinocytes from P1 (one-third of the total amount of T-flas cells). The rest of the cells were frozen as previously described. ${ }^{10}$ From a primary culture, we obtained 3 CAOMEs of oral mucosa, $25 \mathrm{~cm}^{2}$ each, in 30 days. To avoid keratinocyte differentiation we 


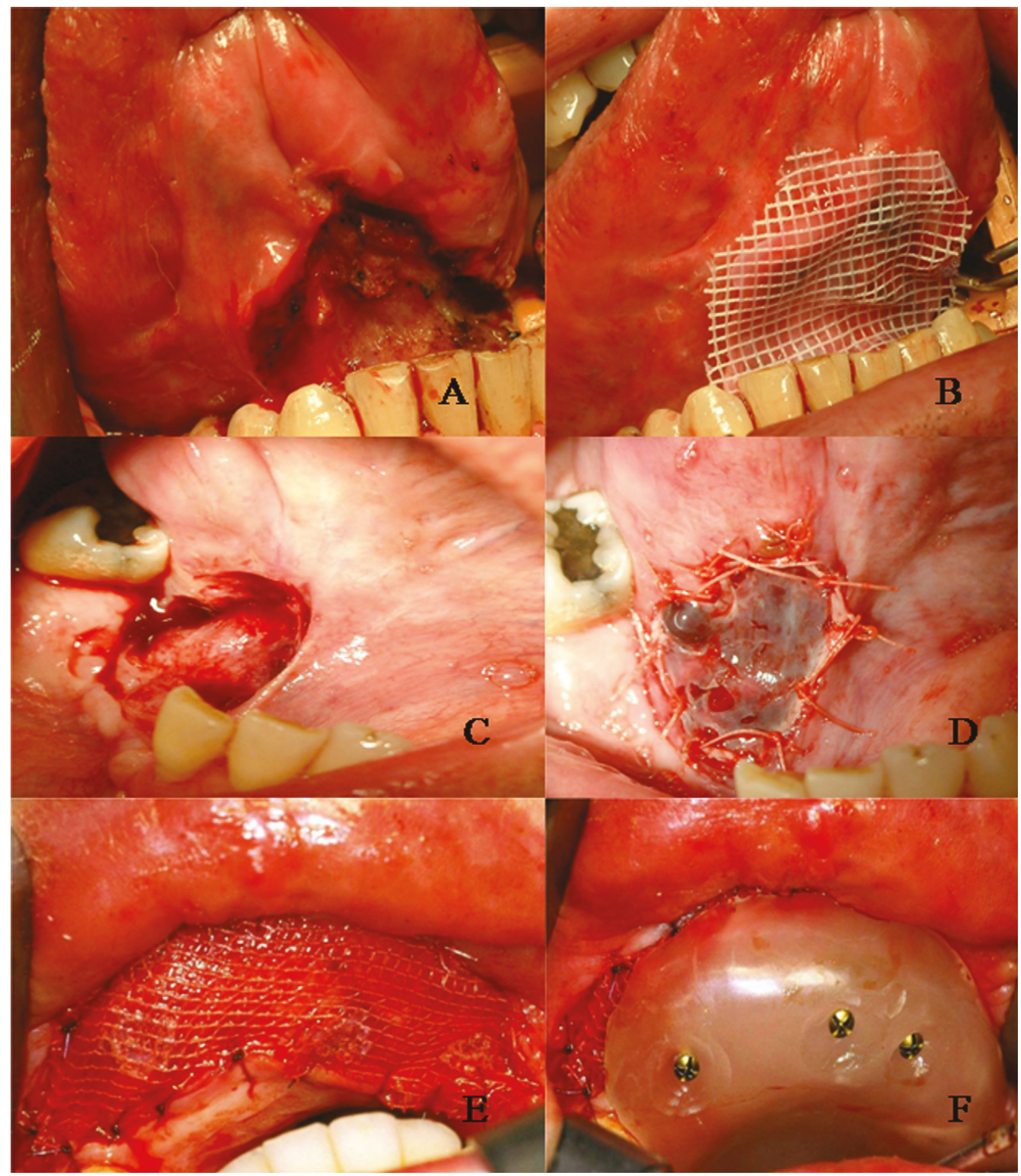

Fig. 2. Surgery and placement of the complete autologous oral mucosa equivalent (CAOME) in patients 1 (A, B), 2 (C, D), and $3(\mathbf{E}, \mathbf{F})$. In cases 1 and 2, the scar bridle was removed from the tongue, and remanent motility was assessed by traction. In case 2, the gauze cover was not kept, and this weakened the CAOME. In case 3, the labial fla of the alveolar rim could be removed, thus expanding the vestibular depth. Afterward, the CAOME was covered with a previously crafted acrylic splint.

used the submerged method. The handling of CAOME became easier following the method previously described by Meana et al. ${ }^{11}$

\section{Patient selection}

Four patients that required a CAOME for reconstructive purposes were selected. They were each given a 
Table II. Scale by Ewers et al., ${ }^{16}$ which shows the analyzed parameters and the degree of improvement achieved based on the outcomes of those parameters

\begin{tabular}{lllll}
\hline & \multicolumn{4}{c}{ Degree of improvement } \\
\cline { 2 - 5 } \multicolumn{1}{c}{ Parameter } & \multicolumn{1}{c}{ None } & \multicolumn{1}{c}{ Mild } & Good & Excellent \\
\hline Tongue motility & Poor motility & Reaches alveolar ridge & Reaches labial vermilion & Reaches all areas of the lips \\
Phonation & Unintelligible & Almost intelligible & Intelligible & Normal \\
Alveolar ridge height & Without keratinized mucosa & $<5 \mathrm{~mm}$ & $5-10 \mathrm{~mm}$ & $>10 \mathrm{~mm}$ \\
\hline
\end{tabular}

detailed explanation of the procedure, and their written informed consents were obtained. The study was approved by the Regional Clinical Research Ethics Committee of the Princedom of Asturias and was guided by the Helsinki Declaration principles.

Patients 1 and 2 had undergone surgery owing to an epidermoid carcinoma in the ventral surface of the tongue (case 1) or in the oral floo (case 2). The reconstruction of the defect was performed with direct closure at the alveolar mucosa. In the late postoperative period, these patients developed a lingual ankylosis which prevented them from carrying out a normal life and constituted a major speech impediment. Patients 3 and 4 required keratinized mucosa to reconstruct the alveolar rim. Patient 3 underwent surgery owing to a pleomorphic adenoma in the upper maxilla; the alveolar rim was covered with a labial mucosa flap reducing the depth of the oral vestibule, which in turn reduced the prosthetic space available. Patient 4 came to our practice to treat a wide epulis fissuratu located in the anterior face of the mandibular alveolar rim. To minimize the loss of soft tissues after extirpation, the application of a CAOME was proposed after the resection of the lesion during the same operation. Because the specifi keratinized pattern of CAOME should be consistent with that of the original mucosal tissue sample, biopsies for patients 1 and 2 were taken from the cheek and for patients 3 and 4 from healthy gum (tuberosity). Clinical statuses are summarized in Table I and illustrated in Fig. 1.

\section{CAOME grafting}

In cases 1 and 2, the tongue was freed from the alveolar rim; it was tested (via traction) that it could reach beyond the lower labial vermilion. After careful hemostasis, the CAOME was sculpted following the shape of the recipient area. Handling was performed with gauze bound to the equivalent with Histocryl. Once the CAOME was correctly adapted to the site, suture was applied with Vicryl 4/0; in case 2, the gauze was removed. In case 3 , the labial mucosa fla was released from the alveolar rim, and a CAOME was applied over the defect in bare cortical bone. To ensure the protection of the CAOME, an acrylic splint was built and fixe to the bone, covering the equivalent, with $6-\mathrm{mm}$
Table III. Outcomes after applying the scale by Ewers et al. ${ }^{16}$ both before surgery and one month after complete autologous oral mucosa equivalent grafting (before/after)

\begin{tabular}{cclc}
\hline Patient & TM & Phonation & \multicolumn{1}{c}{ ARH } \\
\hline 1 & Mild/good & Mild/excellent & \\
2 & Mild/mild & Mild/good & \\
3 & & & None/good \\
4 & & & $<5 \mathrm{~mm} / \mathrm{mild}$ \\
\hline
\end{tabular}

$T M$, Tongue motility; $A R H$, alveolar ridge height.

titanium screws (Walter-Lorenz, Spain). Finally, in patient 4 , after resecting the epulis fissuratum a CAOME was applied with no splint covering it. All patients received an antibiotic treatment for 7 days consisting of amoxicillin-clavulanate $(875 \mathrm{mg} / 8 \mathrm{~h}$, oral $)$ together with nonsteroidal antiinflammatorie (ibuprofen, 600 $\mathrm{mg} / 8 \mathrm{~h}$, oral). Interventions are illustrated in Fig. 2.

\section{Patient follow-up and assessment}

The following parameters were monitored every 48 hours during the firs week: graft loss, presence of edema, bleedings, signs of local infection, graft necrosis or contraction, and any other symptoms patients might mention. The stitches, gauze, and surgical splint were removed 1 week after surgery.

To assess the presence or absence of clinical improvement compared with the status before CAOME application, and because the classical approach for the treatment of these defects is a skin or autologous mucosa graft, the scale designed by Ewers et al. ${ }^{12}$ was used (Table II). It evaluates 3 parameters: tongue motility, phonation, and amount of alveolar gum achieved vertically (measured from where the marginal gum would be to the mucogingival line). Data for evaluation were gathered both before CAOME grafting and 1 month after surgery.

\section{RESULTS}

A certain amount of hyperemia was observed in the target area during the immediate postoperative period, which was more intense in the borders of the graft. No signs of infection, hematoma, or bleeding were present.

At 1 week after surgery, the gauze was removed and the presence of the graft was asserted, featuring 


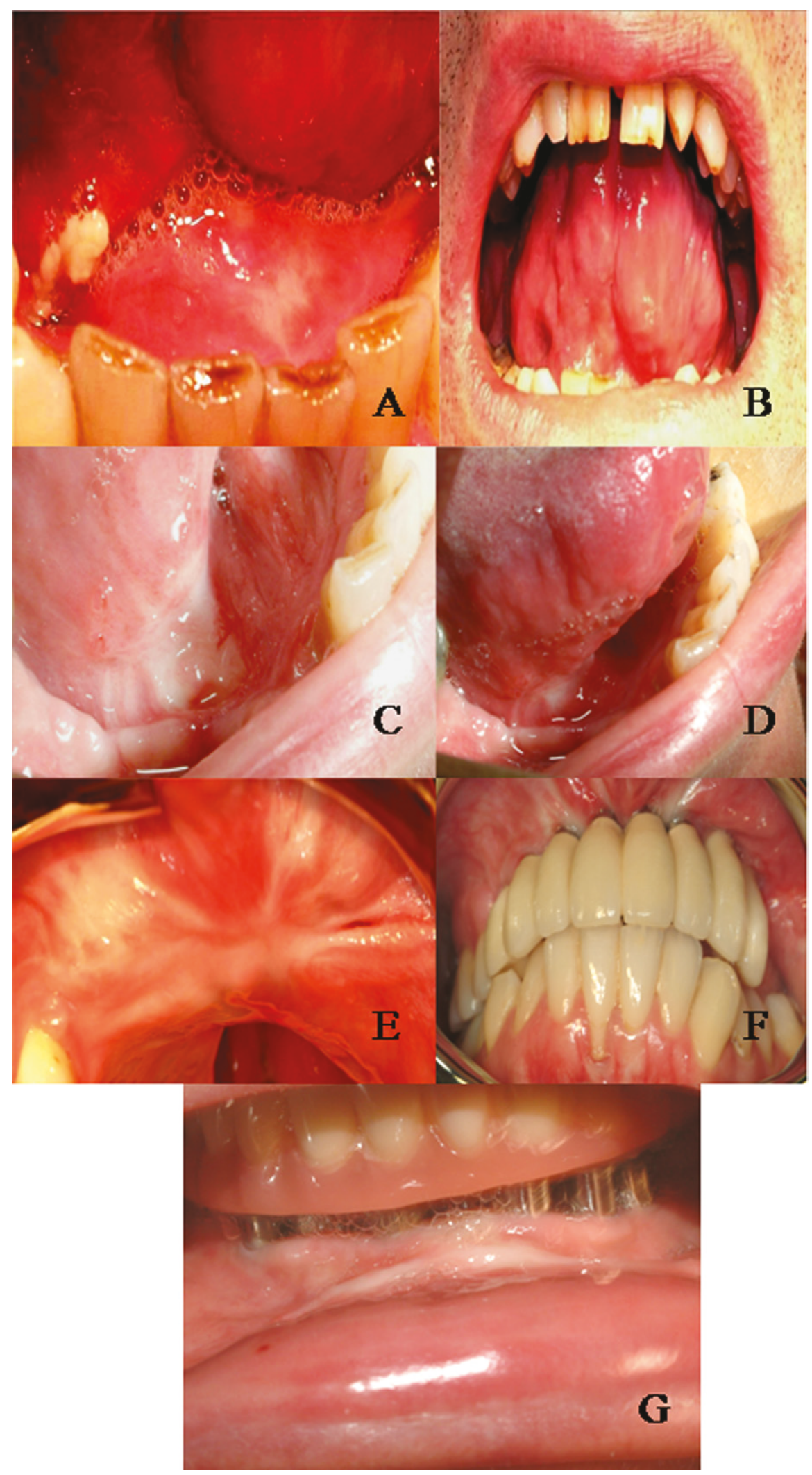

Fig. 3. Outcomes at 1 month after CAOME grafting in patients 1, 2, and 3 (A-F). Patient 1 recovered lingual motility, and his phonation capabilities improved (B). Note the absence of the previous scar bridle and the perfect reconstruction of the lingual ventral side and the lingual root (A). In patient 2, we managed to remove the scar bridle (C), although the functional 
a strong binding with the subjacent tissue. However, we did observe some amount of contraction, which was especially noticeable in the periphery of the graft. This was more evident in patient 2, whose CAOME had not been covered with gauze. During the second week, we noticed that hyperemia was all but gone in most part of the intervened area, although a slight difference was still present between the grafted area and the periphery. By the third week, the appearance was excellent, and after 1 month the grafted area was effectively indistinguishable from the neighboring tissue.

Patients did not experience any pain during the immediate postoperative period, and their impressions ranged from good to excellent regarding the improvements over their situation before receiving the equivalent. One month after surgery, the scale developed by Ewers et al. ${ }^{12}$ was applied. The degree of sequelae gathered from each patient before CAOME grafting and the outcome after grafting are shown in Table III.

Patient 1 could reach even beyond his labial vermilion, although he couldn't reach all the lip zones. Clinically, the improvement that the patient himself found to be more significan was his phonation capability, which according to our assessment evolved from "mild" to "normal." The phonation capability of patient 2 improved from "mild" to "good." However, even though he could move his tongue toward his hard palate, he couldn't reach the labial vermillion, although he could surpass the limit of the lower alveolar rim. According to the scale by Ewers et al., ${ }^{12}$ the outcome was considered to be "mild," even if propulsion was better than before surgery. In case 3 , not only was it possible to add keratinized mucosa to the alveolar rim, but also a 6-mm height was achieved, together with a thickness similar to that of the surrounding gum and an increase of the vestibular depth. Four months after CAOME grafting, the osseointegrated implants were placed with no complications. As for patient 4, although an increase of the vestibular depth was not achieved, it was possible to preserve the status before surgery; thus, rehabilitation was possible by the use of implants at 5 months after CAOME grafting with no complications. The outcomes of the 4 patients are shown in Fig. 3.

\section{DISCUSSION}

In the present study, we treated the loss of oral mucosa of 4 patients with the use of tissue engineering techniques. These 4 cases are representative of the most common clinical situations in which grafting a CAOME may restore or get close to the premorbid status of the patient.

To assess patient postoperative outcome, and because partial skin grafting or autologous mucosa grating are the treatments of choice, we applied the scale designed by Ewers et al. ${ }^{12}$ both before surgery and 1 month after. Our aim was twofold: to determine as objectively as possible whether any clinical improvement actually took place and to assess whether our technique could reduce the healing time reported in the literature when no medium is used to cover the defect.

It is proven that the complete coverage and healing of a defect without tissue addition does not occur until 60 days after surgery. ${ }^{13}$ Similarly, it is also true that if the coverage of the defect is performed with mucosa equivalents created by tissue engineering, in which only the part belonging to the chorion is brought in, reepithelization does not take place until after 40-45 days. ${ }^{8,14}$ In our cases, the observed results are in keeping with those obtained by other authors. ${ }^{7,8,14}$ Although we assume that this favorable evolution is a consequence of the graft used, no histologic confirmatio of the new epithelium was performed in any of our cases. The only way to verify the presence of the grafted keratinocytes at the surgical site and to confir the evolution of the equivalent in vivo would be to mark the cells in advance and to perform a weekly biopsy of the center of the CAOME for 1 month. Currently, the only reliable marker available is bromodeoxyuridine (BrdU), which integrates itself into cellular DNA and can then be detected by anti-Bdru antibodies. ${ }^{7,15}$ The clinical use of this technique is, in our opinion, rather delicate, because this marker may alter cellular DNA.

Therefore, performing biopsies during the firs month and studying them with hematoxylin-eosin is the only valid method accepted in the literature ${ }^{8,14}$ to check the evolution of the graft and to assess whether that development is due to the added cells. In our study, the applicable biopsies were not performed owing to the

improvements were not as good as with patient 1 (D). In patient 3, the vestibular depth was expanded (E-F), thus enabling prosthetic reconstruction by osseointegrated implants. Finally, in patient $4(\mathbf{G})$ we were able to preserve the existing keratinized gum prior to lesion resection and to achieve prosthetic restoration by osseointegrated implants, although we did not manage to expand the vestibular depth as with patient 3 . 
limitations imposed by the Ethics Committee. We could compare our outcomes only from a macroscopic and clinical perspective.

The macroscopic evolution of the graft during the firs 4 weeks was the same as that reported by other authors who used complete oral mucosa equivalents, ${ }^{7,8,14}$ although we did not fin the remarkable contraction which takes place during the early stages in the cases mentioned by Sauerbier et al., ${ }^{7}$ who used collagen as scaffold for the equivalent.

The hyperemia observed in our patients in the early days after grafting, more intense at the edges, is also mentioned by other authors ${ }^{14}$; they explain it as a consequence of the onset of vascularization, a vital event for the survival of the graft in its early stages. Contrary to other studies, ${ }^{8,14}$ the behavior of our equivalent after removing the gauze 1 week after surgery manifested the presence of a strong binding with the subjacent tissue. At 15 days after surgery, the graft was indistinguishable from the surrounding tissue, and after 1 month, the appearance of the surgical site was excellent, with no visible edges whatsoever.

There is a very small number of clinical cases in the literature about the outcomes of this kind of techniques and, on top of that, very few authors use international scales to assess the results. By applying the scale by Ewers et al. ${ }^{12}$ and comparing our results with those mentioned by Lauer et al. ${ }^{15}$ and Sauerbier et al., ${ }^{7}$ we can conclude that they are indeed similar. However, the results referred by these authors were gathered only after equivalent grafting; no data were collected before surgery, so we do not know about the conditions before grafting. In addition, those results were assessed between 3 and 6 months after grafting, ${ }^{7}$ or even after 3 years, ${ }^{15}$ whereas we gathered the data 1 month after surgery.

Although the standard procedure requires graft compression during the firs week after application to improve its adhesion to the surgical site, this was not necessary in our cases, owing to the good adherence of fibri to the surgical defect; it was enough to use the transport gauze of the CAOME, except in case 3, in which we used an acrylic splint. In case 2 the gauze was removed, and we think that was the reason the results were not as good as in case 1, i.e., the absence of some minimal protection.

In our opinion, the contribution of one of the healing process components, such as fibrin may further a rapid integration into the subjacent tissue, ensuring an early binding and avoiding graft loss. Other authors also support this opinion. ${ }^{16}$

The clinical outcomes of our study, together with those of the other mentioned authors, recommend in vitro-generated oral mucosa equivalents as an alterna- tive to be taken into account for the reconstruction of the oral mucosa; studies are required that compare these techniques with the classic methodologies, thus allowing us to defin the actual and true advantages and disadvantages of cell cultures. ${ }^{17}$

\section{CONCLUSION}

CAOME engineering with the described technique seems to allow for the reconstruction of oral mucosa defects with a predictable, minimally aggressive and completely autologous approach, restoring the premorbid status of the patient with a good degree of approximation. However, it is necessary to perform more conclusive statistical studies to confir our hypotheses.

\section{REFERENCES}

1. Nishida K, Yamato M, Hayashida Y, Watanabe K, Yamamoto K, Adachi E, et al. Corneal reconstruction with tissue engineered cell sheets composed of autologous oral mucosal epithelium. N Engl J Med 2004;351:1187-96.

2. Ang LPK, Nakamura T, Inatomi T, Sotozono C, Koizumi N, Yokoi N, et al. Autologous serum-derived cultivated oral epithelial transplants for severe ocular surface disease. Arch Ophthalmol 2006;124:1543-51.

3. Inatomi $\mathrm{T}$, Nakamura $\mathrm{T}$, Koizumi N, Sotozono C, Yokoi N, Kinoshita S, Kinoshita S. Midterm results on ocular surface reconstruction using cultivated autologous oral mucosal epithelial transplantation. Am J Ophthalmol 2006;141:267-75.

4. Feinberg SE, Aghaloo TL, Cunningham LL Jr. Role of tissue engineering in oral and maxillofacial reconstruction: finding of the 2005. AAOMS research summit. J Oral Maxillofac Surg; 63:1418-25.

5. Iida T, Takami Y, Yamaguchi R, Shimazaki S, Harii K. Development of tissue-engineered human oral mucosa equivalent based on an acellular allogeneic dermal matrix: a preliminary report of clinical application to burn wounds. Scand J Plast Reconstr Surg Hand Surg 2005;39:138-46.

6. Lauer G, Schimming R, Frankenschmidt A. Intraoral wound closure with tissue engineered mucosa: new perspectives for urethra reconstruction with buccal mucosa grafts. Plast Reconstr Surg 2001;107:25-33.

7. Sauerbier S, Gutwald R, Wiedmann-Al-Ahmad M, Lauer G, Schmelzeisen R. Clinical application of tissue-engineered transplants. Part I: mucosa. Clin Oral Implants Res 2006; 17:625-32.

8. Hotta T, Yokoo S, Terashi H, Komori T. Clinical and histopathological analysis of healing process of intraoral reconstruction with ex-vivo produced oral mucosa equivalent. Kobe J Med Sci 2007;53:1-14.

9. Peña I, Junquera LM, Meana A, García E, de Vicente JC, García V. In vitro engineering of complete autologous oral mucosa equivalents: characterization of a novel scaffold. J Periodont Res 2010;45:375-80.

10. Llames SG, Del Rio M, Larcher F, García E, García M, Escamez MJ, Jorcano JL, et al. Human plasma as a dermal scaffold for the generation of a completely autologous bioengineered skin. Transplantation 2004;77:350-5.

11. Meana A, Iglesias J, Madrigal B, Sanchez J. Use of cyanoacrylate glue to prepare cultured keratinocyte sheets for grafting. Burns 1997;23:645-6.

12. Ewers R, Hoffmeister B. Reconstruction of the mandibular den- 
ture bearing area and freeing of the tongue after tumor surgery. J Oral Maxillofac Surg 1988;46:272-5.

13. Ugurlu K, Hüthüt I, Özçelik D, Özer K, Sakiz D, Yildiz K, et al. Epithelization process of free fascial flap used in reconstruction of oral cavity mucosa defects in dogs. Plast Reconstr Surg 2004;113:915-25.

14. Izumi K, Feinberg SE, Iida A, Yoshizawa M. Intraoral grafting of an ex vivo produced oral mucosa equivalent: a preliminary report. Int J Oral Maxillofac Surg 2003;32:188-97.

15. Lauer G, Schimming R. Tissue-engineered mucosa graft for reconstruction of the intraoral lining after freeing the tongue: a clinical and immunohistologic study. J Oral Maxillofac Surg 2001;59:169-77.
16. Martin P. Wound healing: aiming for perfect skin regeneration. Science 1997;276:75-81.

17. Assael LA. The promise of tissue engineering. J Oral Maxillofac Surg 2003;61:155-6.

\section{Ignacio Peña}

Department of Oral and Maxillofacial Surgery

C/Celestino Villamil $\mathrm{s} / \mathrm{n}$

33006 Oviedo (Asturias)

Spain

napegomaxilo@gmail.com 